\title{
Abnormality Detection in Indian ECG using Correlation Techniques
}

\author{
Shahanaz Ayub \\ BIET, Jhansi \\ Jhansi, UP, India
}

\author{
J P Saini \\ MMEC, Gorakhpur \\ Gorakhpur, UP, India
}

\begin{abstract}
The paper proposes a method based on signal processing correlation technique to find out whether the ECG is normal or abnormal. Many of the abnormal ECGs are called Arrhythmias. ECG (lead II) obtained from conventional ECG machine of Indian patients are digitized and the data are crosscorrelated with the reference standard normal ECG data. Two different beats of the same ECG data are also correlated. The correlation parameters are used to identify the ECG as normal or abnormal. The accuracy obtained in this method is $100 \%$. The cross-correlation is done using MATLAB 7.12.0 (R2011a) tools.
\end{abstract}

\section{General Terms}

Correlation

\section{Keywords}

Arrhythmia, Cross-correlation, ECG, Lead II

\section{INTRODUCTION}

Electrical activity of the heart is called ECG i.e. Electrocardiogram. Few of the abnormal ECGs are called Arrhythmia. Most commonly occurring Arrhythmias are Tachycardia, Asystole, Bigeminy, Ventricular premature beats, Fusion beats etc. ECGs are analysed by the physicians and interpreted depending upon their experience. The interpretation may vary by physician to physician. Heart disease is the most common cause of death in the world and the ECG is the most preliminary test to diagnose the heart related problems [1], [2]. The experience based analysis by physicians gives different interpretations of the same ECG if the morphological disturbance becomes somewhat complex as in case of fusion beats. So there is a need to develop a procedure to analyze the ECG automatically especially in rural areas where the ECG machines are available but experienced physicians who could interpret the ECG properly are not available.

Many methods have been tried to analyze the ECG automatically through software like Neural Networks, Digital Signal Processing, FFTs and Wavelet Transforms. Most of them have used the standard ECG MIT-BIH database. In this paper a method is proposed where the data used is extracted digital data from the ECG strip of Indian patients obtained from any conventional ECG machine. The samples digitized at $3.387 \mathrm{~ms}$ (i.e. nearly $295 \mathrm{~Hz}$ sampling frequency) are used.

Many works have been done in this area and Artificial Neural Networks give highest accuracy but the drawback of this method is that it requires a huge database to train the networks [1], [2]. Hence in this paper a method is proposed which uses correlation techniques using MATLAB tools. The ECG beat is taken as a signal. The ECG beat consists of 155 samples, i.e. 54 at left from $\mathrm{R}$ peak and 100 samples at right from $\mathrm{R}$ peak. So that in a beat, complete information of start of $\mathrm{P}$ wave till end of $\mathrm{T}$ wave is available.

\section{OBJECTIVE OF THE WORK}

The objective of this work in targeted towards the rural community, so that the rural patients could be diagnosed for the heart problems in less time as well as more accurately, so that the physicians have the primary information about the heart disorders and could start a treatment early.

\section{METHODOLOGY}

Correlation technique has been used to decide whether the ECG is normal or abnormal. A total of 34 records are analyzed through correlation. A reference ECG signal is chosen to correlate the other 33 ECG signals. The reference ECG taken is of 25 years old Indian male, Jitendra Bahadur Maurya and named as Normal Record 1(NR1).

The first step is to digitize all the ECG paper records and store the digital values. Then for each record 155 samples are taken such that it comprises one ECG beat. 54 samples are taken at left and 100 at right of the R peak sample. For each record samples for 2 beats are taken and stored. 1st beat is first correlated with the beat of reference ECG and then with its 2nd beat. Correlation parameters and correlation percentage are then calculated. The overall method is summarized in figure 1 .

\subsection{Digital Data Extraction}

The ECG of Indian patients were taken by BPL 108-T machine which is very commonly used in India. The lead II ECG recorded on ECG paper is scanned at 300dpi using hp Laser Jet 3020 and the images are stored in JPEG format. The desired portion for which digital data is needed is cropped with the help of image tools. Here we select 3 beats for every patient. One ECG beat starts from $\mathrm{P}$ wave and ends to the T wave.

The selected ECG image which contains 3 beats is then converted to gray image. The background grids are removed by thresholding the gray image. The digital data is extracted from this thresholded image which contains only the ECG waveform as black pixels and white background using MATLAB tools [3] - [5]. Figure 2 shows the scanned desired portion of ECG and figure 3 shows the plot obtained by the data extracted from scanned image of record NR1. 


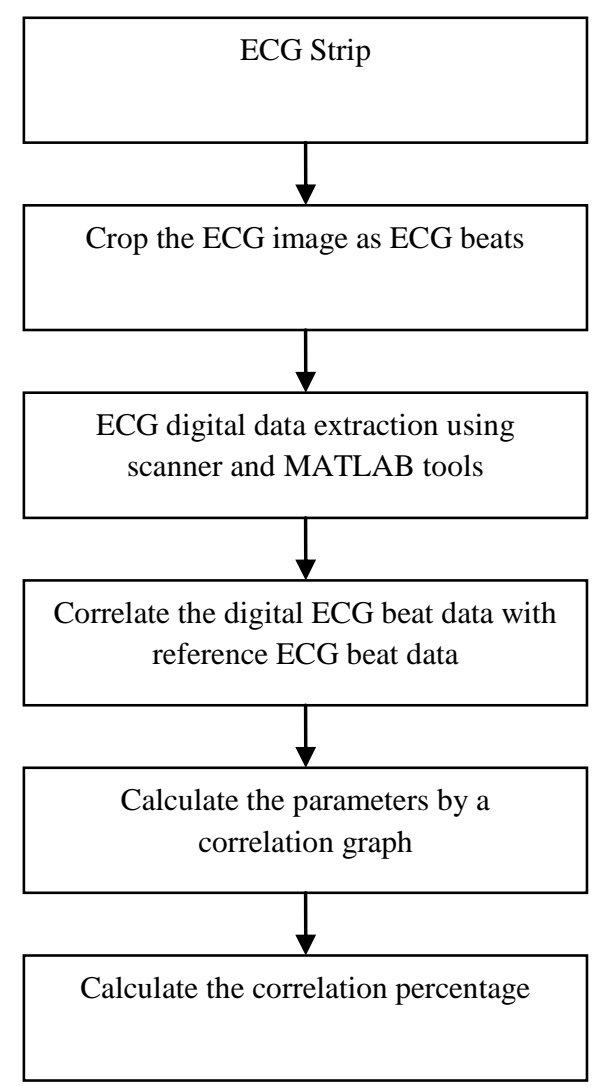

Fig. 1 Methodology

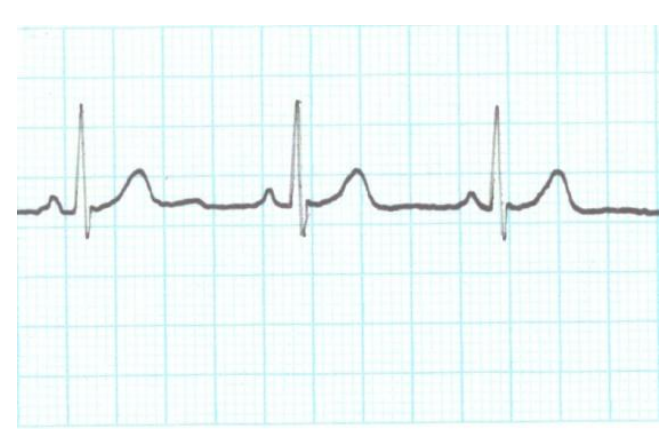

Fig. 2 Scanned ECG of record NR1

\subsection{Calculation of Correlation Parameters and Percentage}

\section{Cross-correlation}

It is used to find out similarity or relationship between the signals $x(n)$ and $y(n)$ delayed by arbitrary delayed factor $K$. $y(n-K)$ is the signal delayed by factor $K$. $n$ is the number of samples in the sequence [6], [7]. Cross-correlation function for discrete time signals $\mathrm{x}(\mathrm{n})$ and $\mathrm{y}(\mathrm{n}-\mathrm{K})$ is given by (1).

$R_{x x}(\mathrm{~K})=\frac{1}{N} \sum_{n=0}^{N-1} x(n) y(n-\mathrm{K})$

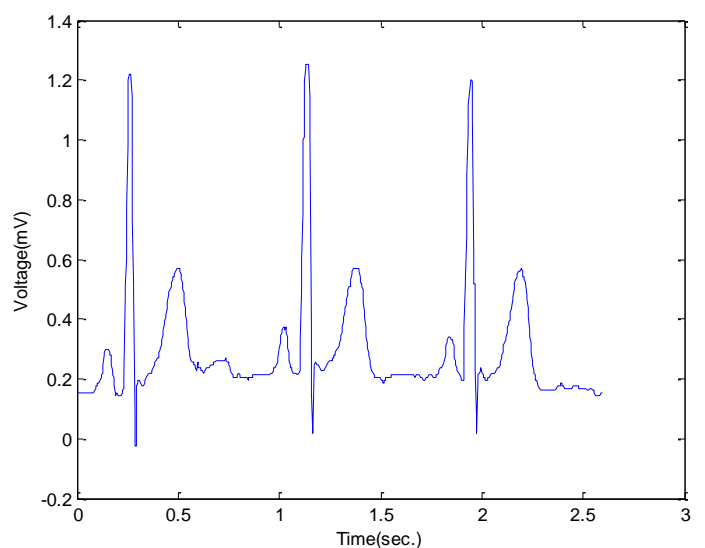

Fig. 3 Plot of extracted data of record NR1

If $R_{x x}(0)$ is the cross-correlation function of input $x$ and $x$ at zero time lag, $R_{x y}(\mathrm{~K})$ is the cross-correlation function of input $x$ and $y$ at $\mathrm{K}^{\text {th }}$ time lag, then the normalized crosscorrelation function, $\rho_{x y}(\mathrm{~K})$ is given by (2) [6].

$\rho_{x y}(\mathrm{~K})=\frac{R_{x y}(\mathrm{~K})}{\sqrt{\left(R_{x x}(0)\right) \times\left(R_{y y}(0)\right)}}$

Range of normalized cross-correlation function is:

$|0 \leq| \rho_{x y}(\mathrm{~K})|\leq 1|$

\section{Auto-correlation}

It is used to find out similarity or relationship between the signals $x(n)$ and signal $x(n)$ delayed by arbitrary delayed factor K. Signal delayed by factor K is $x(n-\mathrm{K}), \mathrm{N}$ is the number of samples in the sequence [6], [7]. Auto-correlation function for discrete time signals $x(n)$ and $x(n-\mathrm{K})$ is given by (4).

$R_{x x}(\mathrm{~K})=\frac{1}{N} \sum_{n=0}^{N-1} x(n) x(n-\mathrm{K})$

If $R_{x x}(0)$ is the auto-correlation function at zero time lag, $R_{x x}(\mathrm{~K})$ is the auto-correlation function at $\mathrm{K}^{\text {th }}$ time lag, then the normalized auto-correlation function, $\rho_{x x}(\mathrm{~K})$ is given by (5) [6].

$\rho_{x x}(\mathrm{~K})=\frac{R_{x x}(K)}{R_{x x}(0)}$

Range of normalized auto-correlation function is:

$|0 \leq| \rho_{x x}(\mathrm{~K})|\leq 1|$ 


\section{Correlation Parameters}

When any ECG beat is correlated with the reference ECG beat, a curve is obtained known as correlation curve.

Figure 4 shows an Auto-correlation curve for NR1.

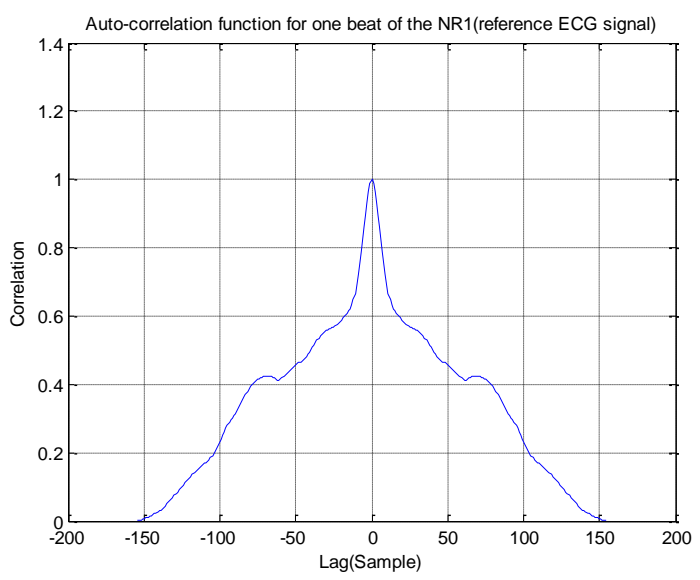

Fig. 4 Auto-correlation curve for NR1

Some parameters are calculated from that curve, which support the analysis. The parameters are MNCC, Min1, Max1, $\mathrm{k}$ (Min1) and $\mathrm{k}(\mathrm{Max} 1)$. MNCC is defined as the Maximum Normalized Cross Correlation function at zero lag. Min1 is defined as amplitude at the first minima in the positive lag. Max1 is defined as the amplitude at the first maxima in the positive lag. k (Min1) is the delay of side lobe Min1 and k (Max1) is the delay of side lobe Max1 in terms of lags (samples) [7].

\section{Correlation Coefficient}

Correlation is the phenomena to established relation between two variables. It may be positive relationship, negative relationship or no relationship. If one variable increases as the other variable increases then a positive relationship is there. If one variable increases as the other variable decreases then it is a negative relationship. Correlation shows relationship between two variables. But it does not show how strong the relationship is. A single number which determine how strong the relationship between two variables or how closely one variable related to other variable we use correlation coefficient [5], [8], [9].

The following mathematical formula is used to compute the correlation coefficient between $X$ and $Y$, where, $X$ and $Y$ are matrices or vectors of the same size.

$r=\frac{\sum_{m} \sum_{n}\left(X_{m n}-\bar{X}\right)\left(Y_{m n}-\bar{Y}\right)}{\sqrt{\left(\sum_{m} \sum_{n}\left(X_{m n}-\bar{X}\right)^{2}\right)\left(\sum_{m} \sum_{n}\left(Y_{m n}-\bar{Y}\right)^{2}\right)}}$

\section{Coefficient of Determination}

To find out the similarity of one variable to other variable in percentage, the square of correlation coefficient i.e. $r^{2}$ is calculated which is known as coefficient of determination. Correlation percentage is the percentage of coefficient of determination [6].

Correlation $(\%)=r^{2} \times 100$

\section{RESULTS}

The test beats and their correlation curves are shown in following figures 5-10. The tables 1,2 and 3 are the calculation results

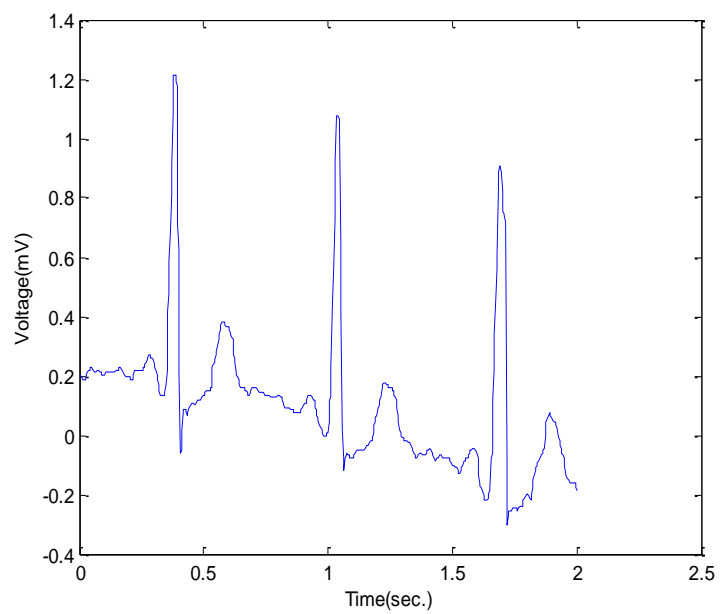

Fig. 5(a) ECG trace of NR2

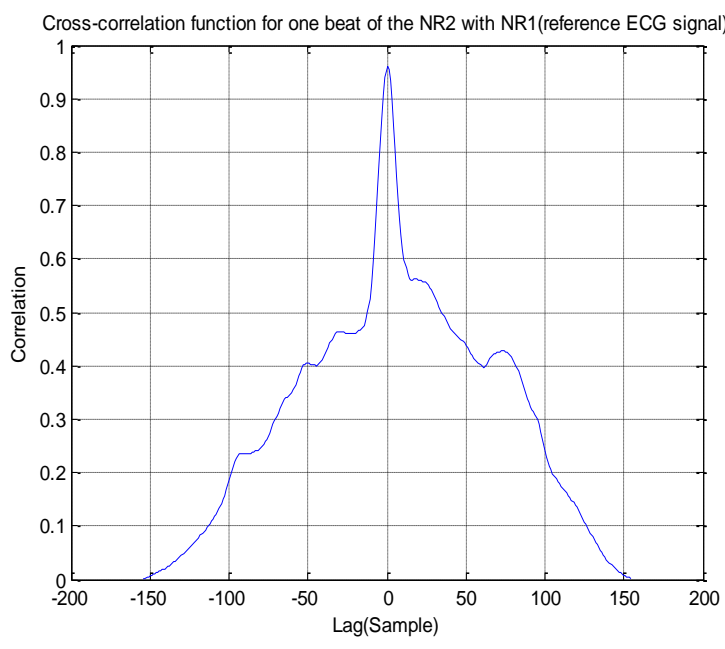

Fig. 5(b) Correlation of NR2 with NR1

Where, $\bar{X}=$ mean of $X$ and $\bar{Y}=$ mean of $Y$ 


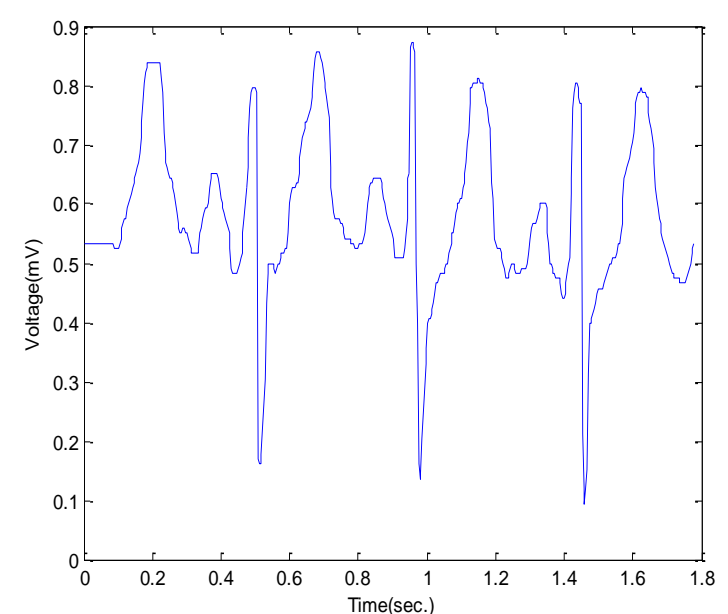

Fig. 6(a) ECG trace of AR1

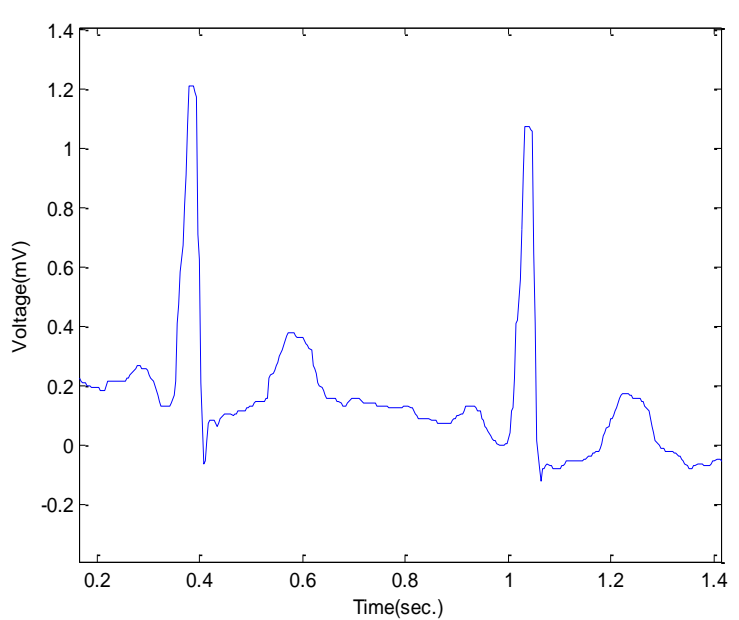

Fig. 7(a) ECG trace of NR2 showing its $1^{\text {st }}$ and $2^{\text {nd }}$ beat

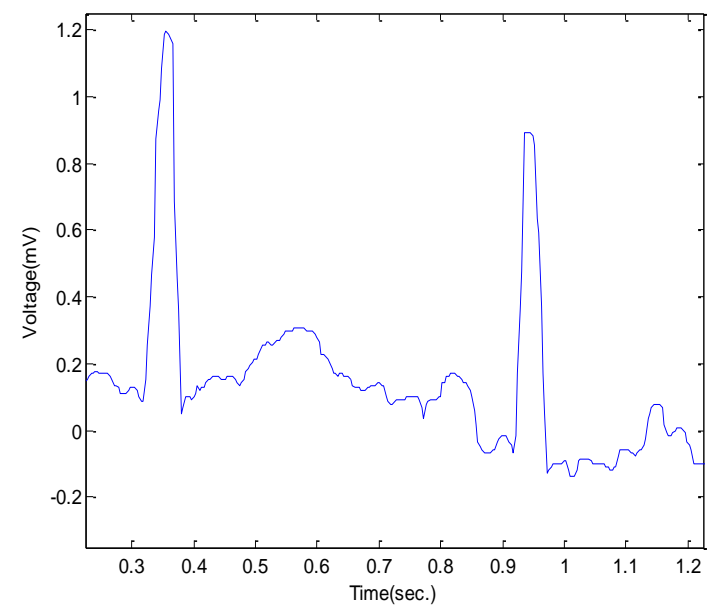

Fig. 8(a) ECG trace of AR2 showing its $1^{\text {st }}$ and $2^{\text {nd }}$ beat

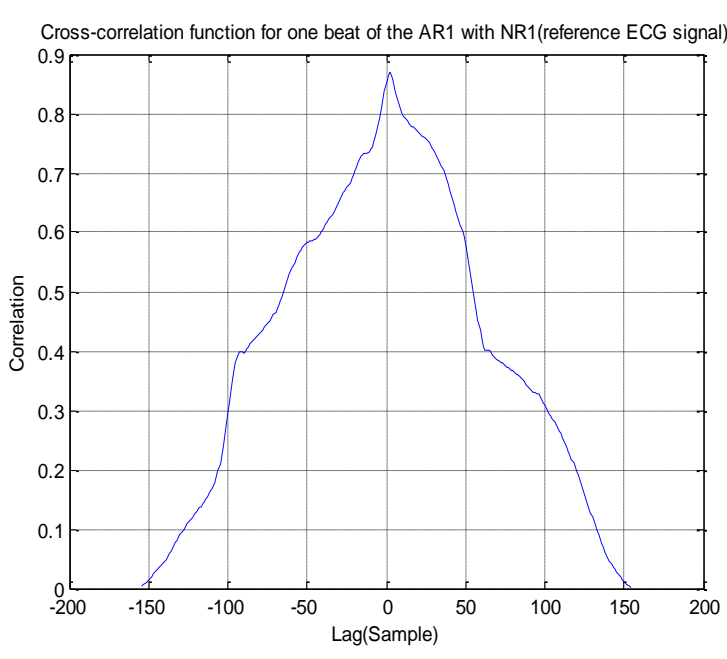

Fig. 6(b) Correlation of AR1 with NR1

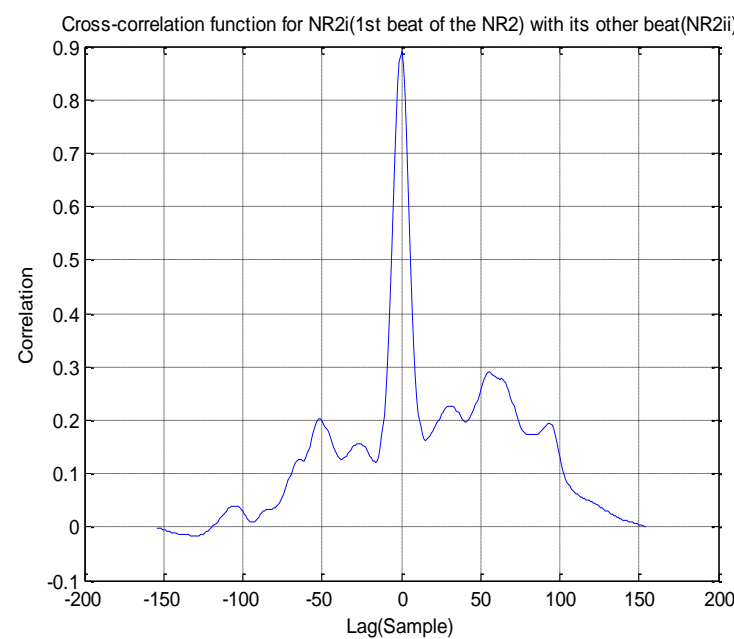

Fig. 7(b) Correlation of NR2i with NR2ii

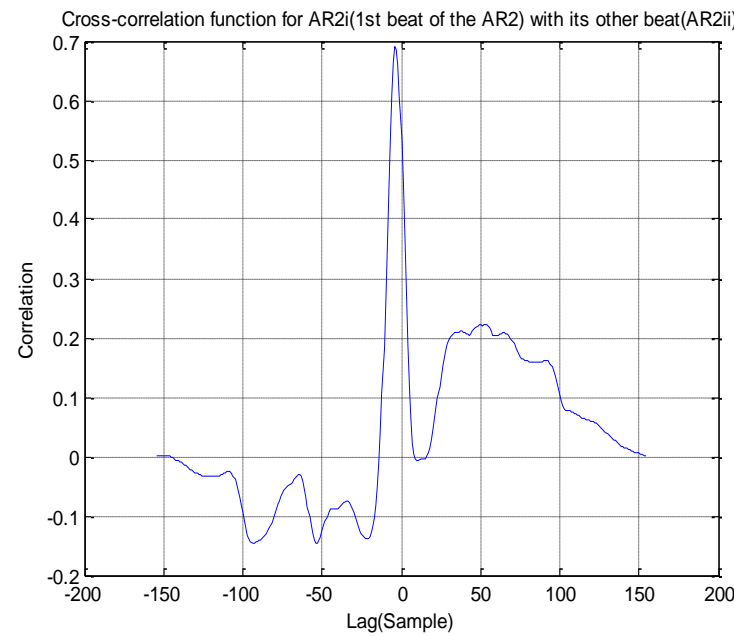

Fig. 8(b) Correlation of AR2i with AR2i 


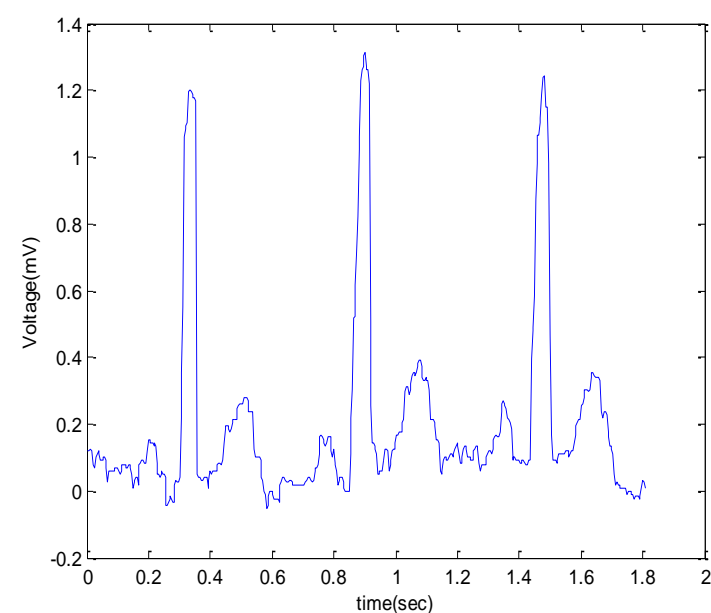

Fig. 9(a) ECG trace of AR19

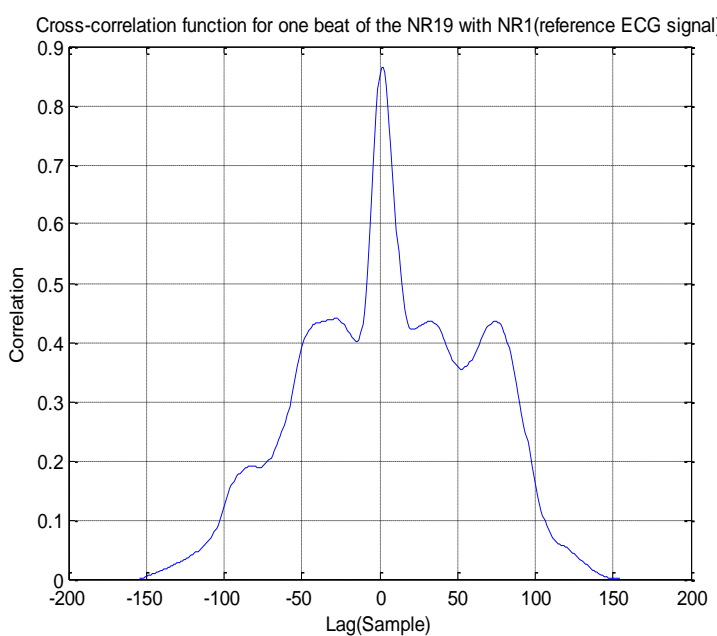

Fig. 9(b) Correlation of NR19 with NR1

Table 1 Correlation Parameters and Correlation Percentage

\begin{tabular}{|c|c|c|c|c|c|c|}
\hline Record & MNCC & Min1 & k(Min1) & Max1 & k(Max1) & Correlation (\%) \\
\hline NR1 \& NR1 & 1.0 & 0.411742 & 61 & 0.422507 & 69 & 100 \\
\hline NR1 \& NR2 & 0.95926 & 0.560701 & 16 & 0.562513 & 18 & 84.9232 \\
\hline NR1 \& AR1 & 0.854583 & - & - & 0.869016 & 2 & 8.4809 \\
\hline NR2i \& NR2ii & 0.888635 & 0.163106 & 15 & 0.227305 & 31 & 97.2485 \\
\hline AR2i \& AR2ii & 0.532318 & -0.00638 & 10 & 0.211268 & 38 & 38.0870 \\
\hline NR1 \& NR19 & 0.854398 & 0.421147 & 21 & 0.434765 & 32 & 60.0994 \\
\hline
\end{tabular}

\section{INFERENCES}

In this paper ECG beat is considered as test cycle. No R-R interval is taken as test cycle as if the heart beat varies i.e. $72 \mathrm{bpm}$ or $76 \mathrm{bpm}$; though it is a normal ECG because of different bpms the RR position will vary which will give poor correlation. Hence the test cycle is an ECG beat (54 samples at left of RR peaks and 100 samples at right of peaks) which include start of $\mathrm{P}$ wave end of $\mathrm{T}$ wave. As seen from the table MNCC is not a parameter which decides the ECG as normal or abnormal as for (NR1 \& AR1) case it is 0.854583 which is similar to that of normal ECG. In this study and referring table 2, correlation percentage above $60 \%$ indicate the ECG as normal. So correlation percentage is the main criteria to decide whether the ECG is normal or abnormal and after that the other parameter are considered. Because of varying nature of ECG it is difficult to get a symmetric correlation curve though the ECG is normal. Hence Min1 and Max1 are the parameters which are considered to support our study, from the study and referring table 1 negative value or no value of Min1 is an indication of abnormal ECG.
Table 2 Correlation Percentage for normal records

\begin{tabular}{|c|c|}
\hline Normal Record No. & Correlation (\%) \\
\hline NR1 & 100 \\
\hline NR2 & 84.9232 \\
\hline NR3 & 70.3737 \\
\hline NR4 & 76.8601 \\
\hline NR5 & 64.0981 \\
\hline NR6 & 64.4523 \\
\hline NR7 & 69.8946 \\
\hline NR8 & 74.8454 \\
\hline NR9 & 78.3005 \\
\hline NR10 & 80.5946 \\
\hline NR11 & 71.5037 \\
\hline NR12 & 79.6395 \\
\hline NR13 & 66.5424 \\
\hline NR14 & 77.7459 \\
\hline
\end{tabular}




\begin{tabular}{|c|c|}
\hline NR15 & 66.9619 \\
\hline NR16 & 79.4704 \\
\hline NR17 & 73.7528 \\
\hline NR18 & 87.465 \\
\hline NR19 & 60.0994 \\
\hline NR20 & 85.1644 \\
\hline NR21 & 85.7473 \\
\hline NR22 & 78.2264 \\
\hline NR23 & 66.5246 \\
\hline NR24 & 71.6169 \\
\hline NR25 & 74.0014 \\
\hline NR26 & 72.1357 \\
\hline NR27 & 75.7588 \\
\hline NR28 & 83.87 \\
\hline NR29 & 70.4712 \\
\hline
\end{tabular}

Table 3 Correlation Percentage for abnormal records

\begin{tabular}{|c|c|}
\hline Abnormal Record No. & Correlation (\%) \\
\hline AR1 & 8.4809 \\
\hline AR2 & 38.087 \\
\hline AR3 & 19.7651 \\
\hline AR4 & 43.8435 \\
\hline AR5 & 55.3186 \\
\hline
\end{tabular}

\section{CONCLUSION}

Based on above study, correlation analysis is the simplest method to find out whether the ECG is normal or abnormal and also it is easy to implement unlike other studies, result are showing $100 \%$ accuracy, the study is thus useful in rural area where physicians are less trained to comment on ECGs.

\section{REFERENCES}

[1] Shahanaz Ayub, J. P. Saini, 'ECG classification and Abnormality Detection using Cascade Forward Neural Network" in International Journal of Engineering, Science \& Technology, Vol. 3, No. 3, pp. 41-46, 2011.

[2] Shahanaz Ayub, J. P. Saini, 'Fusion Beats extraction from ECG using Neural Network based soft computing Techniques', published in International Journal of Advances in Applied Science Research', Coden (USA), 1(2), pp 76-83, 2010.

[3] Jalel Chebil, Jamal Al-Nabulsi, Mohammed Al-Maitah, "A Novel Method for Digitizing Standard ECG Papers", Proceedings of the International Conference on Computer and Communication Engineering, IEEE, pp.1308-1312, 2008.

[4] Sucharita Mitra, M Mitra, "An Automated Data Extraction System from 12 Lead ECG Images", Computer Methods and Programs in Biomedicine, (Elsevier Science publication), vol. 71(1), pp 33-38, May 2003.

[5] MATLAB Simulink Help, The MathWorks, Inc., MATLAB 7.12.0 (R2011a).

[6] The Radical Statistician: A Beginners Guide to Unleashing the Power of Applied Statistics in the Real World (5th Ed.) Jim Higgins Publishing. 2006.

[7] Alias Bin Ramli and Putri Aidawati Ahmad "Correlation Analysis for Abnormal ECG Signal Features Extraction", 4th National Conference on Telecommunication Technology Proceedings, Shah Alam, Malaysia, IEEE Proceedings, pp. 232-237, 2003.

[8] Chuang-Chien Chiu, Tong-Hong Lin and Ben-Yi Liau "Using Correlation Coefficient in ECG Waveform for Arrhythmia Detection", Biomedical Engineering Applications, Basis \& Communications, Vol. 17 No. 3, pp. 37-42, June 2005.

[9] Feng Zhao, Qingming Huang, Wen Gao, "Image Matching By Normalized Cross-Correlation", ICASSP, IEEE Proceedings, pp. 729-732, 2006. 